\title{
The fascial system and exercise intolerance in patients with chronic heart failure: hypothesis of osteopathic treatment
}

This article was published in the following Dove Press journal:

Journal of Multidisciplinary Healthcare

30 October 2015

Number of times this article has been viewed

\author{
Bruno Bordoni ${ }^{1-3}$ \\ F Marelli ${ }^{2,3}$ \\ 'Don Carlo Gnocchi Foundation, \\ Department of Cardiology, IRCCS \\ Santa Maria Nascente, Milan, Italy; \\ ${ }^{2}$ School CRESO, Osteopathic Centre \\ for Research and Studies, Falconara \\ Marittima, AN, Italy; ${ }^{3}$ School CRESO, \\ Osteopathic Centre for Research and \\ Studies, Castellanza, VA, Italy
}

\begin{abstract}
Chronic heart failure is a progressive, debilitating disease, resulting in a decline in the quality of life of the patient and incurring very high social economic costs. Chronic heart failure is defined as the inability of the heart to meet the demands of oxygen from the peripheral area. It is a multi-aspect complex disease which impacts negatively on all of the body systems. Presently, there are no texts in the modern literature that associate the symptoms of exercise intolerance of the patient with a dysfunction of the fascial system. In the first part of this article, we will discuss the significance of the disease, its causes, and epidemiology. The second part will explain the pathological adaptations of the myofascial system. The last section will outline a possible osteopathic treatment for patients with heart failure in order to encourage research and improve the general curative approach for the patient.
\end{abstract}

Keywords: manual therapy, fatigue, chronic heart failure, osteopathic

\section{Introduction}

Chronic heart failure (CHF) is a progressive, debilitating disease, resulting in a decline in the quality of life of the patient and incurring very high social economic costs. ${ }^{1}$ There is no single clinical trial which is able to diagnose CHF. A physical examination of the patient by the physician and a medical history presenting symptoms such as congestion and/or hypoperfusion of the organs are used to make a diagnosis. ${ }^{1} \mathrm{CHF}$ is defined as the inability of the heart to meet the demands of oxygen from the peripheral area. It is a multi-aspect complex disease which impacts negatively on all of the body systems. ${ }^{2}$ This patient population often has a low quality of life, and the majority of patients have a short life expectancy, with a strong chance of dying within 5 years from diagnosis. ${ }^{3}$ Current data estimate that 5.8 million people in North America are suffering from this disease, and approximately 23 million people worldwide are diagnosed with a CHF dysfunction. However, considering that the average age of the population is rising and, thanks to advances in the medical treatment for cardiovascular diseases, the mortality rate from acute diseases is reducing, the number of patients diagnosed with CHF is expected to increase in the coming years. ${ }^{4}$ Fatigue and exercise intolerance are the most obvious clinical manifestations in patients with CHF. Initially, medical practice considered these events to occur as the result of a decreasing perfusion to the musculature and, consequently, the stimulation of metabolic acidosis in the area. ${ }^{5}$ Research and repeated clinical evaluations have shown that these symptoms progress regardless of the hemodynamic situation to the musculature, pointing any scientific investigation toward other possible causes. ${ }^{6}$ There is a discrepancy between the exercise intolerance and the values of the frequency of ejection (the value showing the function
Correspondence: Bruno Bordoni Foundation Don Carlo Gnocchi IRCCS, Department of Cardiology, Institute of Hospitalization and Care with Scientific Address, S Maria Nascente, Via Capecelatro 66, Milan 20100, Italy Tel +390234963006I7

Email bordonibruno@hotmail.com 
of the left ventricle). ${ }^{5}$ A nonphysiological adaptation of the muscle appears to be the most important cause for symptoms of patients with $\mathrm{CHF}^{2}$

The so-called "muscle hypothesis" is based on the fact that the peripheral muscles send an altered afferent to the central nervous system, with a return of pathological efferents to the cardiovascular system. ${ }^{7}$ These anomalies seem to be initially induced by a reduced blood flow to the fibers, with a neurohormonal overactivation negatively impacting the entire body system.

It was shown that greater change in the phenotype of muscle cells from an aerobic metabolism (MHC1) to an anaerobic metabolism (MHC2a and MHC2b), when correlated to fatigue in these patients, resulted in greater exercise intolerance. ${ }^{5}$ Patients with CHF show earlier muscular acidosis and greater phosphocreatine depletion compared with normal controls. ${ }^{6}$ There is evidence that inflammatory mediators affect this metabolic change in patients diagnosed with CHF, although the exact mechanism by which the proinflammatory cytokines act on the muscle fibers is not clear.

Increased levels of tumor necrosis factor (TNF- $\alpha$ ) and interleukin (IL)-1 $\beta$ are seen in patients with CHF. Inflammation aggravates skeletal muscle cell apoptosis and ubiquitindependent proteolysis. ${ }^{6}$ It has been reported that CHF patients show resistance to or low levels of insulin-like growth factors (IGFs), including IGF-1, which are responsible for low levels of synthesis of growth hormone or GH6. This decrease of GH was related to decreased muscular volume and strength in $\mathrm{CHF}^{6}{ }^{6}$ Levels of catabolic hormones, such as cortisol, catecholamines, and angiotensin II, were significantly increased, whereas the levels of anabolic hormones (testosterone, dehydroepiandrosterone sulfate [DHEAS]) and IGF-1 were decreased. These disorders were shown to relate to muscle wasting, anemia, and the clinical severity and prognosis of CHF patients. ${ }^{6}$

It is likely that an important responsibility can be ascribed to oxidative stress, which is recognized as a start signal that activates the proinflammatory process. ${ }^{6}$ This will cause further nonphysiological afferents to be sent, creating a pathological cycle. ${ }^{7}$ The generation of reactive oxygen species and the peroxidation of lipids are also enhanced in the skeletal muscle in CHF: oxidative stress might participate in accelerated apoptosis and atrophy of skeletal muscle fibers. ${ }^{6}$

Another factor to consider is the intervention of the sympathetic nervous system, because in CHF patients its activity is increased when compared to healthy subjects. Overactivation of the renin-angiotensin system is another important factor in skeletal muscle abnormality in $\mathrm{CHF}^{6}$
When blood demanded during the acute phase is greater than the supply rate of the heart, this involves mechanisms which compensate for this to maintain peripheral perfusion and adequate blood pressure. In this scenario, the sympathetic system plays an important role in coordinating the reninangiotensin-aldosterone system, causing vasoconstriction. Even though these compensatory mechanisms are initially beneficial in the acute phase, they become counterproductive if they persist in the long term. ${ }^{8}$ Inadequate perfusion to exercising muscle is followed by increased reactive oxygen species, triggering muscle inflammation, and could contribute to the skeletal abnormalities in CHF. ${ }^{6}$

The weakness due to the respiratory muscles is another key factor responsible for the symptoms of fatigue and exercise intolerance in patients with CHF. The respiratory muscles undergo a metabolic change, different from the muscle groups of the legs. This increases aerobic metabolism and decreases anaerobic metabolism, with a decrease in contractile respiratory force. ${ }^{9}$ The reasons for this are not fully understood.

Additional factors affect the exercise intolerance, such as undernutrition and poor physical condition. Patients with CHF sometimes have anorexia and malabsorption for psychological reasons or because of the gastrointestinal congestion. ${ }^{6}$

Physiotherapy that involves active physical activity improves symptoms in this patient population. ${ }^{9,10}$ Currently, we do not know if a dysfunction of the fascial system, mainly composed of fibroblasts, contributes negatively to symptoms of fatigue and exercise intolerance in patients with CHF. It is not known if manual fascial therapy and/or osteopathy is able to decrease the fatigue and exercise intolerance in patients with CHF. The literature, however, recognizes fascial dysfunction as a cause in many pathological situations. ${ }^{11}$ The fascial system, if altered, generates a symptomatology that deteriorates the health condition of the patient, who very often develops symptoms that are more significant than the clinical parameters diagnosed through medical diagnostic devices. Chronic fatigue, for instance, can be associated with the fascial system, particularly when the pathological disorder has persisted for several years. A recent experimental study has shown that common physiological mechanisms may be involved in the causation of muscle pain and fatigue; the nociceptive afferent inputs from the fascial system can modulate the afferent response from the central nervous system. If the afferent is not physiological, the efferent will be in dysfunction and in pathology. ${ }^{11}$

In the next section, we will attempt to revise the presence of symptoms in this population of patients with altered 
functions of the fascial system: the rationale is to encourage research to see if, by combining the usual cardiovascular physiotherapy approach with a manual therapy, the symptoms of fatigue and exercise intolerance in patients with CHF can be improved. We will also propose a possible process of osteopathic treatment, based on each of the author's 20 years of clinical experience. The aim of the proposed osteopathic techniques is to release the fascial restrictions and alter the mechanical properties of the fascia in, eg, its density, the hardness of the tissue, and its viscosity, so that the fascia may adapt more quickly to the physical stimulus it receives. ${ }^{12}$ The same objectives are proposed by other non-osteopathic manual fascial techniques. ${ }^{13}$

\section{Fatigue and the fascial system}

Every body structure is wrapped in connective tissue, fascia, creating a structural continuity that gives form and function to every tissue and organ. ${ }^{11}$ The fascial tissue is equally distributed throughout the entire body, enveloping, interacting with, and permeating blood vessels, nerves, viscera, meninges, bones, and muscles, creating various layers at different depths and forming a tridimensional metabolic and mechanical matrix. The fascia becomes an organ that can affect an individual's health. ${ }^{12}$

Muscle fatigue is associated with a decline in motor coordination and performance. ${ }^{14}$ The fascial continuum is essential for transmitting the muscle force and for correct motor coordination: the fascia is a vital instrument that enables the individual to communicate and live independently. The transmission of the force is ensured by the fascial integrity, which is expressed by the motor activity produced. ${ }^{11}$

The fibroblasts, the fascia's foundations, have the ability to change their vector, reflecting the force of the mechanical vector present, making the transmission of the tension produced, or managed, more fluid and ergonomic. The fascial system is rich in proprioceptors, such as the Ruffini and Pacinian corpuscles, particularly in the areas of transition between the fascia and the articulation and between the fascia and the contractile tissue in the muscle. The fascial continuum can be considered to be an organ in the mechanical sense, influencing daily postural patterns. ${ }^{11}$ Chronic fatigue may be associated with a dysfunction of the fascial system, particularly when the dysfunction persists over long periods. ${ }^{11}$ Recent studies have shown that the nociceptive afferents from the fascial system can cause symptoms such as fatigue and muscle pain: a fascial dysfunction sends nonphysiological information to the central nervous system, with a pathological return efferent. ${ }^{11}$ The various layers that make up the fascial system must slide properly over each other, allowing the fascia to perform its physiological functions. ${ }^{11}$ A restriction of the fascia may cause symptoms such as fatigue and motor incoordination. ${ }^{11,15}$ Several researchers have reported benefits when treating the fascial system manually. ${ }^{16-19}$

A manual approach could improve motor coordination with a reduction of metabolic expenditure and a decreased intolerance to exercise, because we know that an alteration of the fascia alters proprioceptors. ${ }^{11}$ It may reduce circulating levels of proinflammatory cytokines. We know that an osteopathic treatment influences the cytokine response, and dysfunction of the fascial system can be the source of these substances: the same substances that cause an inflammatory situation in patients with systemic CHF. ${ }^{6,12,20}$

The manual fascial treatment could affect the intramuscular $\mathrm{pH}$, the value of which is higher in patients with CHF: fibroblasts are adept at modulating acid/base parameters. ${ }^{5,11}$ The accumulation of waste metabolites alter the $\mathrm{pH}$ within the fascia with a more acidic cellular environment. The increase in acidity will cause a fascial dysfunction, reducing slippage of the various layers that constitute the fascial continuum. ${ }^{11}$

It is known that an increase of the contractile cell temperature negatively affects performance, stimulating damage to the integrity of the muscle fiber. ${ }^{21-24}$ The same accumulation of metabolites in the extracellular matrix can affect the temperature values. ${ }^{25}$ In patients with $\mathrm{CHF}$, there is a catabolic picture, which leads to loss of muscle tissue, resulting in fatigue and intolerance to exercise. ${ }^{2,5}$ The likely benefits that would lead to manual fascial treatment in patients with $\mathrm{CHF}$ are related to the function of the fascial system itself. Fibroblasts are capable of modulating the temperature of the extracellular matrix, allowing the removal of waste metabolites and favoring the production of hyaluronic acid, which, by assisting the correct sliding of the various fascial layers, acts as a lubricant and prevents nonphysiological friction and also keeps the temperature under control. ${ }^{12}$

The fibroblasts directly affect contractile tissue repair. They secrete different soluble substances, such as IGFs, fibroblast growth factors (FGFs), hepatocyte growth factor (HGF), ILs, and nitric oxide (NO), as a response to mechanical information undergone by the muscles; they control the differentiation of the myoblasts or precursor muscle cells, orientating their epigenetic response. ${ }^{12}$

The fascial system affects circa the $2 \%$ of a maximal voluntary contraction and circa the $1 \%$ of muscular tone in a state of rest; the muscular tone is the mechanical tension or muscular strength. ${ }^{26}$ This tone is sufficient to handle and properly distribute the bodily oscillations provided by the variation in the respiration and heart rate, to maintain an adequate postural pattern. ${ }^{26}$ In patients with $\mathrm{CHF}$, the lack of coordination and a difficulty 
in walking are highlighted and are probably as a result of alterations of the myofascial system, altering the postural patterns and implementing the way energy is expended. ${ }^{27}$

We can hypothesize that by improving the function of the fascial system through manual treatment, the patient's posture will also improve.

\section{Hypothesis of osteopathic treatment}

The fascial osteopathic technique is the application of a low-load, long-duration stretch into the myofascial complex, intended to restore the optimal length of this complex. ${ }^{12}$ The length of treatment duration is subjective, and is in direct response to the tissue of each individual patient, including the number of sessions. Treatment ends when the tissue treated is soft and without restrictions when treated manually.

The practice of the proposed treatment involves fascial osteopathic techniques, applied to the respiratory diaphragm, the pelvic floor or pelvic diaphragm, and the buccal floor or buccal diaphragm.

When you breathe in, the tongue or buccal diaphragm undergoes electrical activity that precedes the lowering of the respiratory diaphragm and pelvic floor or pelvic diaphragm. ${ }^{28}$ There is a close central and peripheral neurological relationship between the three diaphragms. There is also a link between the fascial continuity of the three diaphragms capable of reciprocally influencing each other's function. ${ }^{28}$ We can strongly hypothesize that by manipulating these three structures, with the ultimate aim of improving their function, the effect on the body as a whole will be positive. ${ }^{29}$ In patients with $\mathrm{CHF}$, the diaphragm is weak, with tense and nonphysiological positioning. ${ }^{9,30}$ Some authors report patients

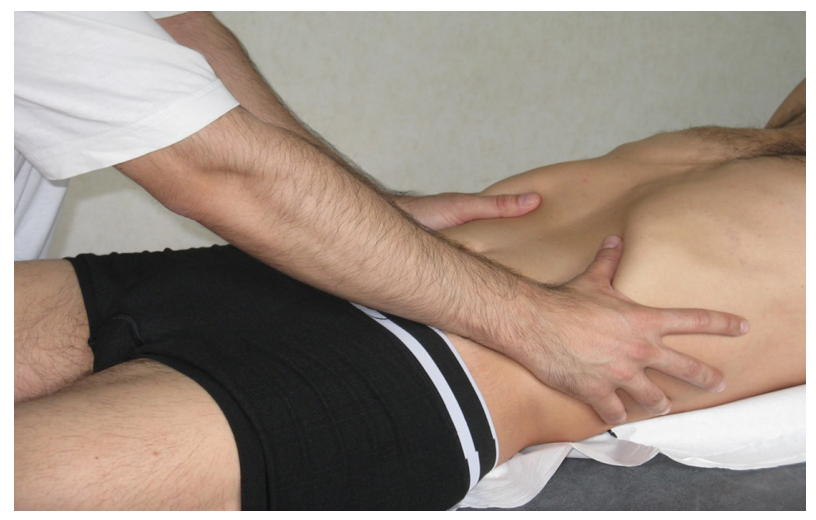

Figure I Treatment of the diaphragm.

Notes: Place the thumbs and the whole tenar side under the diaphragm, in anterolateral position. The purpose is to search for a tensional balance between the right and left hemicupula.

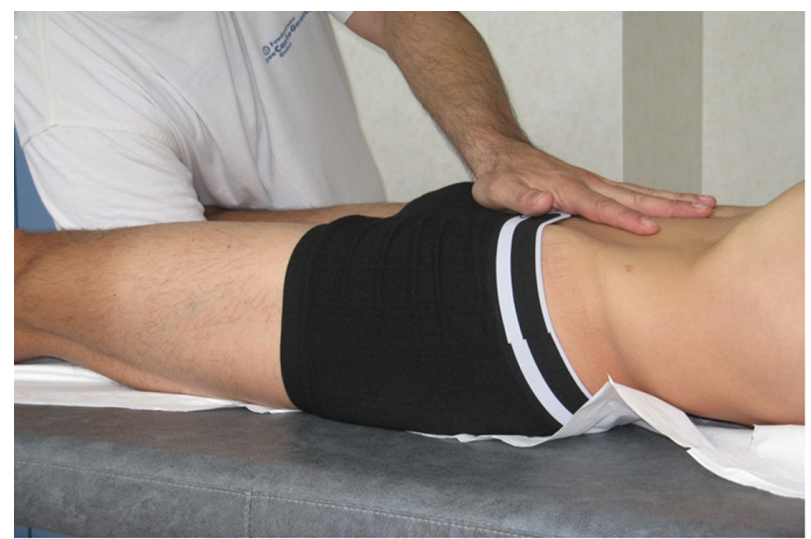

Figure 2 Treatment of the pelvic floor.

Notes: With the patient supine, place one hand under the sacral bone and the other on the pubis, with fingers turned upward, toward the face. When the patient inhales, carefully help the sacral bone rise, while at the same time helping the pubic bone to descend. During exhalation, perform the process in reverse order, until the previous tensions disappear.

with CHF having problems swallowing. ${ }^{31}$ The literature shows that over $50 \%$ of patients with CHF suffer from problems related to urinary incontinence, which is often related to muscle disorders in the pelvic floor. ${ }^{32,33}$

One of the fundamental concepts of fascial osteopathy is the biotensegrity. The biotensegrity is based on the presence of discontinuous compression elements (bones) that balance the stress generated or received by continuous tension elements (muscle and fascia). ${ }^{34}$ This means that rebalancing the overall tensional balance so as to improve the function of the fascial system, affects the whole body system positively. ${ }^{34}$ Working on these interconnected parts of the body in terms of fascial and neurological links; it is possible hypothetically to create an improvement in the patient's symptoms, due to the improvements of the fascial system. Evidence-based medicine is based not only on scientific research highlighted

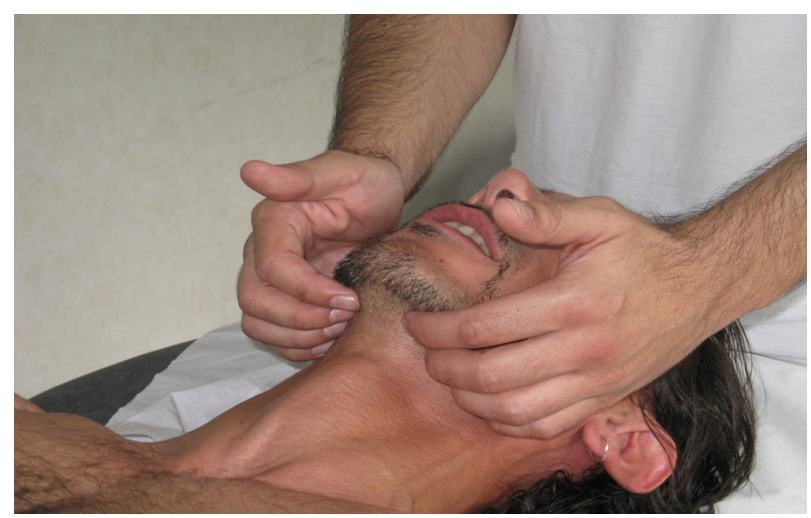

Figure 3 Treatment of the floor of the mouth.

Note: Place the fingertips in a medial position to the jawline and apply uniform pressure on both sides to balance the existing muscular tensions. 
in articles, but also on the patient's experience of treatment and the clinical experience of the operator. ${ }^{35}$

The process starts by treating the respiratory diaphragm (Figure 1), continuing with the pelvic floor (Figure 2), and ending with the floor of the mouth (Figure 3).

\section{Conclusion}

$\mathrm{CHF}$ is a progressive and debilitating disease that results in a decline in the quality of life of the patient, incurring very high social economic costs. A significant skeletal muscular pathology seems to constitute the main underlying mechanism for exercise intolerance.

The modern literature offers no texts associated with the symptoms of exercise intolerance of the patients having a dysfunction of the fascial system. We know that the fascial system may be one of the causes of different local and systemic symptoms when there is a fascial dysfunction, and we know that a manual and osteopathic treatment is able to bring benefit to the function of the fascia. This article has discussed the possibility of a fascial osteopathic approach in patients with CHF, with the aim of improving the symptoms of fatigue. We await further research to see if patients with CHF will derive greater symptomatological benefits by combining the usual physiotherapy approach with a manual fascial therapy.

\section{Disclosure}

The authors report no conflicts of interest in this work.

\section{References}

1. Ramani GV, Uber PA, Mehra MR. Chronic heart failure: contemporary diagnosis and management. Mayo Clin Proc. 2010;85(2):180-195.

2. Tzanis G, Dimopoulos S, Agapitou V, Nanas S. Exercise intolerance in chronic heart failure: the role of cortisol and the catabolic state. Curr Heart Fail Rep. 2014;11:70-79.

3. Siabani S, Driscoll T, Davidson PM, Leeder SR. A randomized controlled trial to evaluate an educational strategy involving community health volunteers in improving self-care in patients with chronic heart failure: rationale, design and methodology. Springerplus. 2014;3:689.

4. Gaggin HK, Januzzi JL Jr. Biomarkers and diagnostics in heart failure. Biochim Biophys Acta. 2013;1832(12):2442-2450.

5. Okita K, Kinugawa S, Tsutsui H. Exercise intolerance in chronic heart failure - skeletal muscle dysfunction and potential therapies. Circ J. 2013;77(2):293-300.

6. Mann DL, Reid MB. Exercise training and skeletal muscle inflammation in chronic heart failure: feeling better about fatigue. J Am Coll Cardiol. 2003;42(5):869-872.

7. Carrington CA, Fisher WJ, Davies MK, White MJ. Is there a relationship between muscle fatigue resistance and cardiovascular responses to isometric exercise in mild chronic heart failure? Eur J Heart Fail. 2001;3(1):53-58.

8. Zucker IH, Xiao L, Haack KK. The central renin-angiotensin system and sympathetic nerve activity in chronic heart failure. Clin Sci (Lond). 2014;126(10):695-706.

9. Cahalin LP, Arena RA. Breathing exercises and inspiratory muscle training in heart failure. Heart Fail Clin. 2015;11(1):149-172.
10. Clark AL. Origin of symptoms in chronic heart failure. Heart. 2006;92(1):12-16.

11. Bordoni B, Zanier E. Clinical and symptomatological reflections: the fascial system. J Multidiscip Healthc. 2014;7:401-411.

12. Bordoni B, Zanier E. Understanding fibroblasts in order to comprehend the osteopathic treatment of the fascia. Evid Based Complement Alternat Med. 2015;2015:860934.

13. Picelli A, Ledro G, Turrina A, Stecco C, Santilli V, Smania N. Effects of myofascial technique in patients with subacute whiplash associated disorders: a pilot study. Eur J Phys Rehabil Med. 2011;47(4):561-568.

14. Emge N, Prebeg G, Uygur M, Jaric S. Effects of muscle fatigue on grip and load force coordination and performance of manipulation tasks. Neurosci Lett. 2013;550:46-50.

15. Bordoni B, Zanier E. Skin, fascias, and scars: symptoms and systemic connections. J Multidiscip Healthc. 2013;7:11-24.

16. Cho SH, Kim SH, Park DJ. The comparison of the immediate effects of application of the suboccipital muscle inhibition and self-myofascial release techniques in the suboccipital region on short hamstring. JPhys Ther Sci. 2015;27(1):195-197.

17. Ajimsha MS. Effectiveness of direct vs indirect technique myofascial release in the management of tension-type headache. J Bodyw Mov Ther. 2011;15(4):431-435.

18. Tozzi P, Bongiorno D, Vitturini C. Low back pain and kidney mobility: local osteopathic fascial manipulation decreases pain perception and improves renal mobility. J Bodyw Mov Ther. 2012;16(3):381-391.

19. Andreoli E, Troiani A, Tucci V, et al. Osteopathic manipulative treatment of congenital talipes equinovarus: a case report. J Bodyw Mov Ther. 2014;18(1):4-10.

20. Licciardone JC, Kearns CM, Hodge LM, Bergamini MV. Associations of cytokine concentrations with key osteopathic lesions and clinical outcomes in patients with nonspecific chronic low back pain: results from the OSTEOPATHIC Trial. J Am Osteopath Assoc. 2012; 112(9):596-605.

21. Haddad DS, Brioschi ML, Arita ES. Thermographic and clinical correlation of myofascial trigger points in the masticatory muscles. Dentomaxillofac Radiol. 2012;41(8):621-629.

22. Dokladny K, Myers OB, Moseley PL. Heat shock response and autophagy - cooperation and control. Autophagy. 2015;11(2): 200-213.

23. Febbraio MA. Does muscle function and metabolism affect exercise performance in the heat? Exerc Sport Sci Rev. 2000;28(4):171-176.

24. Jarmuszkiewicz W, Woyda-Ploszczyca A, Koziel A, Majerczak J, Zoladz JA. Temperature controls the oxidative phosphorylation and reactive oxygen species production through uncoupling in rat skeletal muscle mitochondria. Free Radic Biol Med. 2015;83:12-20.

25. Ito A, Aoyama T, Iijima H, et al. Optimum temperature for extracellular matrix production by articular chondrocytes. Int J Hyperthermia. 2014; 30(2):96-101.

26. Masi AT, Hannon JC. Human resting muscle tone (HRMT): narrative introduction and modern concepts. J Bodyw Mov Ther. 2008; 12(4):320-332.

27. Panizzolo FA, Maiorana AJ, Naylor LH, et al. Gait analysis in chronic heart failure: the calf as a locus of impaired walking capacity. J Biomech. 2014;47(15):3719-3725.

28. Bordoni B, Zanier E. Anatomic connections of the diaphragm: influence of respiration on the body system. J Multidiscip Healthc. 2013;6:281-291.

29. Bordoni B, Zanier E. The continuity of the body: hypothesis of treatment of the five diaphragms. J Altern Complement Med. 2015;21(4):237-242.

30. Caruana L, Petrie MC, McMurray JJ, MacFarlane NG. Altered diaphragm position and function in patients with chronic heart failure. Eur J Heart Fail. 2001;3(2):183-187.

31. Bassi D, Furkim AM, Silva CA, et al. Identification of risk groups for oropharyngeal dysphagia in hospitalized patients in a university hospital. Codas. 2014;26(1):17-27.

32. Tannenbaum C, Johnell K. Managing therapeutic competition in patients with heart failure, lower urinary tract symptoms and incontinence. Drugs Aging. 2014;31(2):93-101. 
33. Newman DK. Pelvic floor muscle rehabilitation using biofeedback. Urol Nurs. 2014;34(4):193-202.

34. Martínez Rodríguez R, Galán del Río F. Mechanistic basis of manual therapy in myofascial injuries. Sonoelastographic evolution control. J Bodyw Mov Ther. 2013;17(2):221-234.
35. Masic I, Miokovic M, Muhamedagic B. Evidence based medicine-new approaches and challenges. Acta Inform Med. 2008;16(4):219-225.

\section{Publish your work in this journal}

The Journal of Multidisciplinary Healthcare is an international, peerreviewed open-access journal that aims to represent and publish research in healthcare areas delivered by practitioners of different disciplines. This includes studies and reviews conducted by multidisciplinary teams as well as research which evaluates the results or conduct of such teams or healthcare processes in general. The journal covers a wide range of areas and welcomes submissions from practitioners at all levels, from all over the world. The manuscript management system is completely online and includes a very quick and fair peer-review system. Visit http://www.dovepress.com/testimonials.php to read real quotes from published authors.

Submit your manuscript here: http://www.dovepress.com/journal-of-multidisciplinary-healthcare-journal 